\title{
THE PERIODIC COMPLEX METHOD IN INTERPOLATION SPACES
}

\author{
ELIRAN AVNI
}

\begin{abstract}
In this paper we consider the "periodic" variant of the complex interpolation method, apparently first studied by Peetre in [7]. Cwikel showed in [5] that using functions with a given period $i \lambda$ in the complex method construction introduced and studied by Calderón (see [4]), one may construct the same interpolation spaces as in the "regular" complex method, up to equivalence of norms. Cwikel also showed in [5] that the constants of this equivalence will, in some cases, "blow up" as $\lambda \rightarrow 0$. We show that the constants of this equivalence approach 1 as $\lambda \rightarrow \infty$. Intuitively, this means that when applying the complex method of Calderón, it makes a very small difference if one restricts oneself to periodic functions, provided that the period is very large.
\end{abstract}

\section{INTRODUCTION}

In this section we recall the basic definitions of interpolation spaces and of the complex method of Calderón and its periodic variant. We also prove several auxiliary claims to be used later in this paper. Most of the definitions in this section appear extensively in the literature (see, e.g., $[1,2,3,6,8]$ ).

Definition 1. Whenever $A_{0}, A_{1}$ are two Banach spaces that are both continuously embedded in some topological Hausdorff vector space $\mathcal{A}$ we say that $\left(A_{0}, A_{1}\right)$ is a Banach couple.

Definition 2. Whenever $\left(A_{0}, A_{1}\right)$ is a Banach couple, we define $A_{0}+A_{1}$ to be the space of all elements $a \in \mathcal{A}$ such that $a=a_{0}+a_{1}$ for some $a_{j} \in A_{j}(j=0,1)$. This is a Banach space, when endowed with the following norm:

$$
\|a\|_{A_{0}+A_{1}}=\inf \left\{\left\|a_{0}\right\|_{A_{0}}+\left\|a_{1}\right\|_{A_{1}}: a_{j} \in A_{j}, j=0,1, a=a_{0}+a_{1}\right\} .
$$

Definition 3. For a given Banach couple $\left(A_{0}, A_{1}\right)$, the statement " $T$ : $\left(A_{0}, A_{1}\right) \rightarrow\left(A_{0}, A_{1}\right)$ is a bounded linear operator" means that $T$

Key words and phrases. Interpolation spaces, periodic complex method.

The research was supported by a Chester and Taube Hurwitz Foundation Fellowship. 
is a linear operator from $A_{0}+A_{1}$ into itself such that the restriction of $T$ to $A_{j}$ is a bounded operator from $A_{j}$ into itself (for $j=0,1$ ).

Remark. We remark that if $T:\left(A_{0}, A_{1}\right) \rightarrow\left(A_{0}, A_{1}\right)$ is a bounded linear operator then automatically $T$ is also a bounded linear operator from $A_{0}+A_{1}$ into itself, and the following inequality holds:

$$
\|T\|_{A_{0}+A_{1} \rightarrow A_{0}+A_{1}} \leq \max \left\{\|T\|_{A_{0} \rightarrow A_{0}},\|T\|_{A_{1} \rightarrow A_{1}}\right\} .
$$

We recall that $A_{0} \cap A_{1}$, when endowed with the norm

$$
\|x\|_{A_{0} \cap A_{1}}=\max \left\{\|x\|_{A_{0}},\|x\|_{A_{1}}\right\},
$$

is also a Banach space. This will be relevant in the following definition.

Definition 4. A Banach space $A$ satisfying $A_{0} \cap A_{1} \subseteq A \subseteq A_{0}+A_{1}$ where all the inclusions are continuous (that is, when the identity maps $i: A_{0} \cap A_{1} \rightarrow A$ and $i: A \rightarrow A_{0}+A_{1}$ are continuous) is called an intermediate space of $\left(A_{0}, A_{1}\right)$.

Definition 5. Whenever $\left(A_{0}, A_{1}\right)$ is a Banach couple, the statement " $A$ is an interpolation space with respect to $\left(A_{0}, A_{1}\right)$ " is a concise way to say the following: $A$ is an intermediate space of $\left(A_{0}, A_{1}\right)$, and the restriction to $A$ of every bounded linear operator $T:\left(A_{0}, A_{1}\right) \rightarrow$ $\left(A_{0}, A_{1}\right)$ is a bounded operator from $A$ into itself.

For future reference, it is convenient to set $\mathbb{S}=\{z \in \mathbb{Z}: 0 \leq \operatorname{Re} z \leq 1\}$. We also denote the interior of $\mathbb{S}$ by $\mathbb{S}^{\circ}$.

Next we define five special spaces, that play an essential role in the complex method and the periodic complex method (cf. [4, 5, 7]). We stress that although the definitions we have given in this paper thus far are applicable for Banach spaces defined over $\mathbb{R}$ or $\mathbb{C}$, from this point onwards we assume all Banach spaces we deal with are defined over $\mathbb{C}$.

Definition 6. The space $\mathscr{F}^{\infty}\left(A_{0}, A_{1}\right)$, sometimes denoted simply by $\mathscr{F}^{\infty}$, consists of all continuous functions $f: \mathbb{S} \rightarrow A_{0}+A_{1}$ that satisfy these conditions:

- The restriction of $f$ to $\{j+i y: y \in \mathbb{R}\}$ is a continuous function into $A_{j}$, for $j=0,1$.

- The restriction of $f$ to $\mathbb{S}^{\circ}$ is an analytic function into $A_{0}+A_{1}$. (One of the equivalent definitions of that statement is that for any bounded linear functional $l: A_{0}+A_{1} \rightarrow \mathbb{C}$, the composition $l \circ f: \mathbb{S}^{\circ} \rightarrow \mathbb{C}$ is an analytic function.)

- $\sup _{z \in \mathbb{S}}\left\{\|f(z)\|_{A_{0}+A_{1}}\right\}<\infty$.

- $\sup _{j=0,1, y \in \mathbb{R}}\left\{\|f(j+i y)\|_{A_{j}}\right\}<\infty$. 
This is a Banach space when endowed with the norm:

$$
\|f\|_{\mathscr{F} \infty}=\sup _{j=0,1, y \in \mathbb{R}}\left\{\|f(j+i y)\|_{A_{j}}\right\} .
$$

Definition 7. The space $\mathscr{F}\left(A_{0}, A_{1}\right)$, or simply $\mathscr{F}$, is the closed subspace of $\mathscr{F}^{\infty}\left(A_{0}, A_{1}\right)$ which consists of functions $f \in \mathscr{F}^{\infty}$ that satisfy $\lim _{|y| \rightarrow \infty}\|f(j+i y)\|_{A_{j}}=0$, for both $j=0$ and $j=1$.

Definition 8. For each fixed $\lambda>0$ the space $\mathscr{F}_{\lambda}\left(A_{0}, A_{1}\right)$, or simply $\mathscr{F}_{\lambda}$, is the closed subspace of $\mathscr{F}^{\infty}\left(A_{0}, A_{1}\right)$ which consists of functions $f \in \mathscr{F}^{\infty}$ that are $i \lambda$-periodic (that is, $f(z)=f(z+i \lambda)$ for all $z \in \mathbb{S}$ ).

Definition 9. Following Calderón in [4], for each $0<\theta<1$, we define $\left[A_{0}, A_{1}\right]_{\theta}=\left\{f(\theta): f \in \mathscr{F}\left(A_{0}, A_{1}\right)\right\}$.

This is a Banach space, when endowed with the norm:

$$
\|a\|_{[\theta]}=\inf \left\{\|f\|_{\mathscr{F} \infty}: a=f(\theta), f \in \mathscr{F}\left(A_{0}, A_{1}\right)\right\} .
$$

Remark. It is well known that in this preceding definition of the space $\left[A_{0}, A_{1}\right]_{\theta}$ one can replace $\mathscr{F}$ by $\mathscr{F}^{\infty}$ and this does not change the set $\left[A_{0}, A_{1}\right]_{\theta}$. Furthermore, replacing $\mathscr{F}$ by $\mathscr{F}^{\infty}$ in (1.1) does not change the norm $\|a\|_{[\theta]}$. This is easily deduced from the fact that for every $f \in \mathscr{F}^{\infty}\left(A_{0}, A_{1}\right)$ and every $\delta>0$, the function $f_{\delta}(z)=e^{\delta(z-\theta)^{2}} f(z)$ is an element of $\mathscr{F}\left(A_{0}, A_{1}\right)$ and from the fact that $\inf _{\delta>0}\left\{\left\|f_{\delta}\right\|_{\mathscr{F} \infty}\right\} \leq$ $\|f\|_{\mathscr{F} \infty}$ (cf., e.g., [5]. This is also implicit in [4].)

An essential feature of the complex method, as proven in [4], is that $\left[A_{0}, A_{1}\right]_{\theta}$ is an interpolation space of $\left(A_{0}, A_{1}\right)$ for any $0<\theta<1$. In other words, given a Banach couple $\left(A_{0}, A_{1}\right)$, Calderón devised a mechanism for constructing a family of interpolation spaces of that couple. We remark that when setting $A_{0}=L^{p_{0}}(\mu)$ and $A_{1}=L^{p_{1}}(\mu)$ one obtains that $\left[A_{0}, A_{1}\right]_{\theta}=L^{p_{\theta}}(\mu)$, where $\frac{1}{p_{\theta}}=\frac{1-\theta}{p_{0}}+\frac{\theta}{p_{1}}$ (with equality of norms) whenever $p_{0}, p_{1} \in[1, \infty]$. This example of spaces of the form $\left[A_{0}, A_{1}\right]_{\theta}$, which of course comes from Thorin's proof of the Riesz-Thorin Theorem, was probably the key motivating factor that guided Calderón in his construction.

Remark. We note that although there are many other examples of Banach couples for which concrete descriptions of the spaces $\left[A_{0}, A_{1}\right]_{\theta}$ may be found, there are also many other examples, including even "natural" ones, where no concrete descriptions of the spaces $\left[A_{0}, A_{1}\right]_{\theta}$ are known.

Definition 10. As in [5], for each $0<\theta<1$ and $\lambda>0$, we define $\left[A_{0}, A_{1}\right]_{\theta}^{\lambda}=\left\{f(\theta): f \in \mathscr{F}_{\lambda}\left(A_{0}, A_{1}\right)\right\}$. 
This is a Banach space, when endowed with the norm:

$$
\|a\|_{[\theta, \lambda]}=\inf \left\{\|f\|_{\mathscr{F} \infty}: a=f(\theta), f \in \mathscr{F}_{\lambda}\left(A_{0}, A_{1}\right)\right\} .
$$

It is easy to see that $\left[A_{0}, A_{1}\right]_{\theta}^{\lambda}$ is an interpolation space of $\left(A_{0}, A_{1}\right)$ for all $0<\theta<1$ and all $\lambda>0$. Furthermore, the inclusion $\left[A_{0}, A_{1}\right]_{\theta}^{\lambda} \subseteq$ $\left[A_{0}, A_{1}\right]_{\theta}$ and inequality $\|a\|_{[\theta]} \leq\|a\|_{[\theta, \lambda]}$ (for all $a \in\left[A_{0}, A_{1}\right]_{\theta}^{\lambda}$ ) are obvious. In fact, Cwikel proved the following theorem in [5]:

Theorem 11. For every $0<\theta<1$ and every $\lambda>0$ we have $\left[A_{0}, A_{1}\right]_{\theta}=$ $\left[A_{0}, A_{1}\right]_{\theta}^{\lambda}$ as two subsets of $A_{0}+A_{1}$. Moreover, there is a $C>0$ such that $\|a\|_{[\theta]} \leq\|a\|_{[\theta, \lambda]} \leq C\|a\|_{[\theta]}$ for all $a \in\left[A_{0}, A_{1}\right]_{\theta}$ (the constant $C$ may depend, in general, on both $\lambda$ and $\theta)$.

Remark. Cwikel also showed in [5] that no uniform upper bound of $C$ (with respect to $\lambda$ ) may be found, since for some Banach couples $\left(A_{0}, A_{1}\right)$, some $\theta \in(0,1)$ and some $a \in\left[A_{0}, A_{1}\right]_{\theta}$ we have

$$
\limsup _{\lambda \rightarrow 0} \frac{\|a\|_{[\theta, \lambda]}}{\|a\|_{[\theta]}}=\infty .
$$

Despite this dependence on $\lambda$ it can be seen (cf. also Theorem 15 below) that for each fixed $\lambda$ the constant $C$ can in fact be bounded independently of $\theta$.

Remark. As mentioned in [5], the periodic complex interpolation spaces can also be defined via consideration of analytic Banach space valued functions defined on an annulus. Let us explain this in a little more detail: For each $\lambda \in(0, \infty)$ we set $\mathbb{A}_{\lambda}=\left\{z \in \mathbb{C}: 1 \leq|z| \leq e^{\frac{2 \pi}{\lambda}}\right\}$. We notice that for each $g: \mathbb{A}_{\lambda} \rightarrow A_{0}+A_{1}$ we can define $f_{g}: \mathbb{S} \rightarrow A_{0}+A_{1}$ by setting $f_{g}(z)=g\left(e^{\frac{2 \pi}{\lambda} z}\right)$. This defines a bijection map between the set of all $A_{0}+A_{1}$-valued functions on $\mathbb{A}_{\lambda}$ and the set of all $A_{0}+A_{1}$-valued functions on $\mathbb{S}$ which are $i \lambda$-periodic. This map enables us to formulate an alternative equivalent definition of $\left[A_{0}, A_{1}\right]_{\theta}^{\lambda}$ by using $A_{0}+A_{1}$-valued functions defined on $\mathbb{A}_{\lambda}$ in an obvious way.

We conclude this section by proving three claims which will be used in the proof of our main result.

Claim 12. Let $\lambda$ be a fixed positive number. Setting $C_{1, \lambda}(\alpha)=e^{\alpha}+$ $2 e^{\alpha-0.25 \alpha \lambda^{2}} \cdot \frac{2-e^{-\alpha \lambda^{2}}}{1-e^{-\alpha \lambda^{2}}}$ for any $\alpha>0$, one has

$$
\sup _{|y| \leq \frac{\lambda}{2}}\left\{\sum_{k \in \mathbb{Z}} e^{\alpha\left(1-(y+k \lambda)^{2}\right)}\right\} \leq C_{1, \lambda}(\alpha) .
$$


Proof. First we shall show that

$$
\sum_{k=1}^{\infty} e^{\alpha\left(1-(y+k \lambda)^{2}\right)} \leq e^{\alpha-0.25 \alpha \lambda^{2}} \cdot \frac{2-e^{-\alpha \lambda^{2}}}{1-e^{-\alpha \lambda^{2}}} .
$$

We notice that for every $k \geq 1$ and $|y| \leq \frac{\lambda}{2}$ we have $y+k \lambda \geq k \lambda-\frac{\lambda}{2} \geq$ $\frac{\lambda}{2}>0$, hence

$$
\begin{aligned}
\sum_{k=1}^{\infty} e^{\alpha\left(1-(y+k \lambda)^{2}\right)} & \leq \sum_{k=1}^{\infty} e^{\alpha\left(1-\left(k \lambda-\frac{\lambda}{2}\right)^{2}\right)} \\
& =\sum_{k=1}^{\infty} e^{\alpha\left(1-\lambda^{2}\left(k-\frac{1}{2}\right)^{2}\right)} \\
& =e^{\alpha}\left(e^{-\alpha\left(\frac{\lambda}{2}\right)^{2}}+\sum_{k=2}^{\infty} e^{-\alpha \lambda^{2}\left(k-\frac{1}{2}\right)^{2}}\right) \\
& \leq e^{\alpha}\left(e^{-\alpha\left(\frac{\lambda}{2}\right)^{2}}+\sum_{k=2}^{\infty} e^{-\alpha \lambda^{2}\left(k-\frac{1}{2}\right)}\right) \\
& =e^{\alpha}\left(e^{-\alpha\left(\frac{\lambda}{2}\right)^{2}}+e^{0.5 \alpha \lambda^{2}} \sum_{k=2}^{\infty} e^{-\alpha \lambda^{2} k}\right) \\
& =e^{\alpha}\left(e^{-\alpha\left(\frac{\lambda}{2}\right)^{2}}+e^{0.5 \alpha \lambda^{2}} \cdot \frac{e^{-2 \alpha \lambda^{2}}}{1-e^{-\alpha \lambda^{2}}}\right) \\
& \leq e^{\alpha}\left(e^{-0.25 \alpha \lambda^{2}}+\frac{e^{-0.25 \alpha \lambda^{2}}}{1-e^{-\alpha \lambda^{2}}}\right) \\
& =e^{\alpha-0.25 \alpha \lambda^{2}} \cdot \frac{2-e^{-\alpha \lambda^{2}}}{1-e^{-\alpha \lambda^{2}}} \cdot
\end{aligned}
$$

This establishes (1.2). Then, replacing $y$ by $-y$ and $k$ by $-k$ in (1.2), we can immediately deduce that for every $|y| \leq \frac{\lambda}{2}$ we also have

$$
\sum_{k=-\infty}^{-1} e^{\alpha\left(1-(y+k \lambda)^{2}\right)} \leq e^{\alpha-0.25 \alpha \lambda^{2}} \cdot \frac{2-e^{-\alpha \lambda^{2}}}{1-e^{-\alpha \lambda^{2}}} .
$$

Finally, when $k=0$ and $|y| \leq \frac{\lambda}{2}$ we may write

$$
e^{\alpha\left(1-(y+k \lambda)^{2}\right)}=e^{\alpha\left(1-y^{2}\right)} \leq e^{\alpha} .
$$


Combining (1.2), (1.3), and (1.4) we obtain

$$
\begin{aligned}
\sup _{|y| \leq \frac{\lambda}{2}}\left\{\sum_{k \in \mathbb{Z}} e^{\alpha\left(1-(y+k \lambda)^{2}\right)}\right\} & \leq e^{\alpha}+2 e^{\alpha-0.25 \alpha \lambda^{2}} \cdot \frac{2-e^{-\alpha \lambda^{2}}}{1-e^{-\alpha \lambda^{2}}} \\
& =C_{1, \lambda}(\alpha)
\end{aligned}
$$

completing the proof.

Claim 13. Let $\lambda$ be a fixed positive number. For every $f \in \mathscr{F}\left(A_{0}, A_{1}\right)$, every $\alpha>0$, every $0<\theta<1$ and every analytic function $g: \mathbb{C} \rightarrow \mathbb{C}$, if $\sup _{z \in \mathbb{S}}\{|g(z)|\}=M<\infty$ then the function $F_{\alpha}: \mathbb{S} \rightarrow A_{0}+A_{1}$ defined by

$$
F_{\alpha}(z)=\sum_{k \in \mathbb{Z}} e^{\alpha(z-\theta+i k \lambda)^{2}} g(z+i k \lambda) \cdot f(z+i k \lambda)
$$

is an element of $\mathscr{F}_{\lambda}\left(A_{0}, A_{1}\right)$. Moreover, we also have

$$
\left\|F_{\alpha}\right\|_{\mathscr{F} \infty} \leq C_{1, \lambda}(\alpha) M \cdot\|f\|_{\mathscr{F} \infty} \text {. }
$$

Proof. It is clear that the scalar valued series $\sum_{k \in \mathbb{Z}} e^{\alpha(z-\theta+i k \lambda)^{2}} g(z+$ $i k \lambda)$ converges absolutely and uniformly in every compact subset of $\mathbb{S}$. This fact implies, first of all, that the $A_{0}+A_{1}$-valued function $F_{\alpha}$ is well defined, continuous, and clearly $i \lambda$-periodic and therefore bounded. The same fact also implies that the restriction of $F_{\alpha}$ to $\mathbb{S}^{\circ}$ is analytic. Defining $\Lambda_{j}=\{j+i y: y \in \mathbb{R}\}$ for $j=0,1$, the fact that the functions $\left.F_{\alpha}\right|_{\Lambda_{j}}: \Lambda_{j} \rightarrow A_{j}$ (for $j=0,1$ ) are continuous (and bounded due to periodicity) follows readily from the same argument.

Thus we have established that $F_{\alpha} \in \mathscr{F}_{\lambda}\left(A_{0}, A_{1}\right)$. Furthermore, bearing in mind that $F_{\alpha}$ is $i \lambda$-periodic, we see that

$$
\left\|F_{\alpha}\right\|_{\mathscr{F} \infty}=\sup _{j=0,1,|y| \leq \frac{\lambda}{2}}\left\{\left\|F_{\alpha}(j+i y)\right\|_{A_{j}}\right\} .
$$

Now, for each fixed $j \in\{0,1\}$ and $|y| \leq \frac{\lambda}{2}$ we have

$$
\begin{aligned}
& \left\|F_{\alpha}(j+i y)\right\|_{A_{j}} \\
= & \lim _{n \rightarrow \infty}\left\|\sum_{k=-n}^{n} e^{\alpha(j+i(y+k \lambda)-\theta)^{2}} g(j+i(y+k \lambda)) \cdot f(j+i(y+k \lambda))\right\|_{A_{j}} \\
\leq & \lim _{n \rightarrow \infty} \sum_{k=-n}^{n}\left|e^{\alpha(j+i(y+k \lambda)-\theta)^{2}} g(j+i(y+k \lambda))\right|\|f(j+i(y+k \lambda))\|_{A_{j}} \\
\leq & \lim _{n \rightarrow \infty} \sum_{k=-n}^{n} e^{\alpha\left((j-\theta)^{2}-(y+k \lambda)^{2}\right)} M\|f\|_{\mathscr{F} \infty} \\
\leq & \lim _{n \rightarrow \infty} \sum_{k=-n}^{n} e^{\alpha\left(1-(y+k \lambda)^{2}\right)} M\|f\|_{\mathscr{F} \infty} .
\end{aligned}
$$


Combining (1.5), (1.6) and Claim 12 we conclude that $\left\|F_{\alpha}\right\|_{\mathscr{F} \infty} \leq$ $C_{1, \lambda}(\alpha) M \cdot\|f\|_{\mathscr{F} \infty}$, as required.

Claim 14. For a given $\lambda>0$ and $0<\theta<1$, let us define $w: \mathbb{C} \rightarrow \mathbb{C}$ by $w(\theta)=1$ and $w(z)=\frac{\lambda}{2 \pi} \cdot \frac{e^{\frac{2 \pi}{\lambda}(z-\theta)-1}}{z-\theta}$ for all $z \neq \theta$. We also define $m(\lambda)=\frac{\lambda}{2 \pi}\left(1+e^{\frac{4 \pi}{\lambda}}\right)$. We then have

$$
\sup _{z \in \mathbb{S}}\{|w(z)|\} \leq m(\lambda) .
$$

Proof. It is easy to see that $w(z)$ is an analytic function in the entire complex plane, bounded on $\mathbb{S}_{2}:=\{z \in \mathbb{C}:-1 \leq \operatorname{Re} z \leq 2\}$. Applying the Phragmén-Lindelöf maximum principle we deduce that

$$
\begin{aligned}
\sup _{z \in \mathbb{S}}\{|w(z)|\} & \leq \sup _{z \in \mathbb{S}_{2}}\{|w(z)|\} \\
& =\sup _{z \in \partial \mathbb{S}_{2}}\{|w(z)|\} \\
& =\sup _{j=-1,2, y \in \mathbb{R}}\{|w(j+i y)|\} .
\end{aligned}
$$

According to the definition of $w(z)$, for $j=-1,2$ and for all $y \in \mathbb{R}$, one has

$$
\begin{aligned}
|w(j+i y)| & =\left|\frac{\lambda}{2 \pi} \cdot \frac{\left(e^{\frac{2 \pi}{\lambda}(j-\theta+i y)}-1\right)}{j-\theta+i y}\right| \\
& \leq \frac{\lambda}{2 \pi|j-\theta|}\left(\left|e^{\frac{2 \pi}{\lambda}(j-\theta+i y)}\right|+1\right) \\
& =\frac{\lambda}{2 \pi|j-\theta|}\left(1+e^{\frac{2 \pi}{\lambda}(j-\theta)}\right) \\
& \leq \frac{\lambda}{2 \pi|j-\theta|}\left(1+e^{\frac{4 \pi}{\lambda}}\right) .
\end{aligned}
$$

Since $j=-1,2$ and $0<\theta<1$ we necessarily have $\frac{1}{|j-\theta|} \leq 1$, and from this (1.7) immediately follows.

\section{The Main Result}

In this section we state and prove our main result which complements the results obtained in [5].

Theorem 15. There is a function $C=C(\lambda)$ such that $\lim _{\lambda \rightarrow \infty} C(\lambda)=$ 1 and, for all Banach couples $\left(A_{0}, A_{1}\right)$, all $0<\theta<1$, all $a \in\left[A_{0}, A_{1}\right]_{\theta}$, and all $\lambda>0$, one has

$$
\|a\|_{[\theta]} \leq\|a\|_{[\theta, \lambda]} \leq C(\lambda)\|a\|_{[\theta]} .
$$

As we have mentioned, the equality $\left[A_{0}, A_{1}\right]_{\theta}=\left[A_{0}, A_{1}\right]_{\theta}^{\lambda}$ was already proven in Theorem 11, and the inequality $\|a\|_{[\theta]} \leq\|a\|_{[\theta, \lambda]}$ obviously 
holds for all $a \in\left[A_{0}, A_{1}\right]_{\theta}=\left[A_{0}, A_{1}\right]_{\theta}^{\lambda}$, so in order to prove Theorem 15 we need only to prove the right hand side of (2.1). The proof extends over the next three subsections:

2.1. A rough estimate. Suppose $x \in\left[A_{0}, A_{1}\right]_{\theta}$, and $f \in \mathscr{F}\left(A_{0}, A_{1}\right)$ is such that $x=f(\theta)$. Let us fix $\lambda>0$ and, following Cwikel in [5], define $G_{\delta}(z)=\sum_{k \in \mathbb{Z}} e^{\delta(z-\theta+i k \lambda)^{2}} w(z+i k \lambda) \cdot f(z+i k \lambda)$ where we leave the value of $\delta>0$ undetermined for now (the function $w(z)$ was defined in Claim 14). Applying Claim 14 and then Claim 13, we see that $G_{\delta} \in \mathscr{F}_{\lambda}\left(A_{0}, A_{1}\right)$ for every $\delta>0$. Moreover, since $w(\theta)=1$ and $w(\theta+i k \lambda)=0$ for all $0 \neq k \in \mathbb{Z}$, we have $G_{\delta}(\theta)=f(\theta)=x$. Again, applying Claims 14 and 13, we gather that

$$
\begin{aligned}
\|x\|_{[\theta, \lambda]} & \leq\left\|G_{\delta}\right\|_{\mathscr{F} \infty} \\
& \leq m(\lambda) C_{1, \lambda}(\delta)\|f\|_{\mathscr{F} \infty} .
\end{aligned}
$$

Finally, taking the infimum over all $f \in \mathscr{F}\left(A_{0}, A_{1}\right)$ such that $x=f(\theta)$ will yield

$$
\|x\|_{[\theta, \lambda]} \leq m(\lambda) C_{1, \lambda}(\delta)\|x\|_{[\theta]}
$$

for all $x \in\left[A_{0}, A_{1}\right]_{\theta}$.

2.2. Some fine tuning. Given an $a \in\left[A_{0}, A_{1}\right]_{\theta}$, for each $f \in \mathscr{F}\left(A_{0}, A_{1}\right)$ such that $a=f(\theta)$ we choose a certain $\rho>0$ to be determined later and define $\tilde{f}_{\rho}(z)=\sum_{k \in \mathbb{Z}} e^{\rho(z-\theta+i k \lambda)^{2}} f(z+i k \lambda)$. According to Claim 13 (when we set $g(z) \equiv 1$ ) we have $\tilde{f}_{\rho} \in \mathscr{F}_{\lambda}\left(A_{0}, A_{1}\right)$. Defining $\tilde{a}=\tilde{f}_{\rho}(\theta) \in\left[A_{0}, A_{1}\right]_{\theta}^{\lambda}$, we have, according to the same claim, that

$$
\begin{aligned}
\|\tilde{a}\|_{[\theta, \lambda]} & \leq\left\|\tilde{f}_{\rho}\right\|_{\mathscr{F} \infty} \\
& \leq C_{1, \lambda}(\rho)\|f\|_{\mathscr{F} \infty} .
\end{aligned}
$$

Defining

$$
P_{n}=\left\|\tilde{a}-\sum_{k=-n}^{n} e^{-\rho(k \lambda)^{2}} f(\theta+i k \lambda)\right\|_{[\theta]}
$$

and

$$
Q_{n}=\left\|\sum_{k=-n}^{n} e^{-\rho(k \lambda)^{2}} f(\theta+i k \lambda)-a\right\|_{[\theta]}
$$

we obviously may write

$$
\|\tilde{a}-a\|_{[\theta]} \leq P_{n}+Q_{n} .
$$

Estimating the expression $Q_{n}$ we get 


$$
\begin{aligned}
Q_{n} & =\left\|\sum_{k=-n}^{n} e^{-\rho(k \lambda)^{2}} f(\theta+i k \lambda)-a\right\|_{[\theta]} \\
& =\left\|\sum_{k=-n}^{n} e^{-\rho(k \lambda)^{2}} f(\theta+i k \lambda)-f(\theta)\right\|_{[\theta]} \\
& \leq \sum_{k=-n}^{-1}\left\|e^{-\rho(k \lambda)^{2}} f(\theta+i k \lambda)\right\|_{[\theta]}+\sum_{k=1}^{n}\left\|e^{-\rho(k \lambda)^{2}} f(\theta+i k \lambda)\right\|_{[\theta]} \\
& \leq \sum_{k=-n}^{-1} e^{-\rho(k \lambda)^{2}}\|f\|_{\mathscr{F} \infty}+\sum_{k=1}^{n} e^{-\rho(k \lambda)^{2}}\|f\|_{\mathscr{F} \infty} \\
& =2 \sum_{k=1}^{n} e^{-\rho(k \lambda)^{2}}\|f\|_{\mathscr{F} \infty} \\
\leq & 2 \sum_{k=1}^{\infty} e^{-\rho k \lambda^{2}}\|f\|_{\mathscr{F} \infty} \\
& =2 e^{-\rho \lambda^{2}} \cdot \frac{1}{1-e^{-\rho \lambda^{2}}}\|f\|_{\mathscr{F} \infty} .
\end{aligned}
$$

We will now show that

$$
\lim _{n \rightarrow \infty} P_{n}=0 .
$$

First we note that, by definition

$$
\lim _{n \rightarrow \infty}\left\|\tilde{a}-\sum_{k=-n}^{n} e^{-\rho(k \lambda)^{2}} f(\theta+i k \lambda)\right\|_{A_{0}+A_{1}}=0 .
$$

Furthermore, we observe that $\left(\sum_{k=-n}^{n} e^{-\rho(k \lambda)^{2}} f(\theta+i k \lambda)\right)_{n \in \mathbb{N}}$ is a Cauchy sequence in $\left[A_{0}, A_{1}\right]_{\theta}$. Indeed, if $m>n$ then

$$
\begin{aligned}
& \left\|\sum_{k=-m}^{m} e^{-\rho(k \lambda)^{2}} f(\theta+i k \lambda)-\sum_{k=-n}^{n} e^{-\rho(k \lambda)^{2}} f(\theta+i k \lambda)\right\|_{[\theta]} \\
\leq & \sum_{k=n+1}^{m}\left\|e^{-\rho(k \lambda)^{2}} f(\theta+i k \lambda)\right\|_{[\theta]}+\sum_{k=-m}^{-n-1}\left\|e^{-\rho(k \lambda)^{2}} f(\theta+i k \lambda)\right\|_{[\theta]} \\
\leq & 2 \sum_{k=n+1}^{\infty} e^{-\rho \lambda^{2} k^{2}}\|f\|_{\mathscr{F} \infty}
\end{aligned}
$$


and since clearly $\lim _{n \rightarrow \infty} 2 \sum_{k=n+1}^{\infty} e^{-\rho \lambda^{2} k^{2}}\|f\|_{\mathscr{F} \infty}=0$, the sequence $\left(\sum_{k=-n}^{n} e^{-\rho(k \lambda)^{2}} f(\theta+i k \lambda)\right)_{n \in \mathbb{N}}$ is Cauchy. This means that there is an element $\bar{a} \in\left[A_{0}, A_{1}\right]_{\theta}$ such that

$$
\lim _{n \rightarrow \infty}\left\|\bar{a}-\sum_{k=-n}^{n} e^{-\rho(k \lambda)^{2}} f(\theta+i k \lambda)\right\|_{[\theta]}=0 .
$$

Since $\|x\|_{A_{0}+A_{1}} \leq\|x\|_{[\theta]}$ for every $x \in\left[A_{0}, A_{1}\right]_{\theta}$ (cf., e.g., [4] paragraph 23 p. 129 where there is implicitly a routine application of the Phragmén-Lindelöf maximum principle for vector valued analytic functions), it is also true that

$$
\lim _{n \rightarrow \infty}\left\|\bar{a}-\sum_{k=-n}^{n} e^{-\rho(k \lambda)^{2}} f(\theta+i k \lambda)\right\|_{A_{0}+A_{1}}=0 .
$$

Combining this last equality with (2.7) we immediately get that $\bar{a}=\tilde{a}$. Using (2.8), we immediately obtain (2.6).

Combining (2.5), (2.6) and (2.4) we get

$$
\|\tilde{a}-a\|_{[\theta]} \leq \frac{2 e^{-\rho \lambda^{2}}}{1-e^{-\rho \lambda^{2}}}\|f\|_{\mathscr{F} \infty} .
$$

2.3. Conclusion. Combining (2.2), (2.3), and (2.9) we see that for each $a \in\left[A_{0}, A_{1}\right]_{\theta}^{\lambda}$ and each $f \in \mathscr{F}\left(A_{0}, A_{1}\right)$ such that $a=f(\theta)$ we may write

$$
\begin{aligned}
\|a\|_{[\theta, \lambda]} & \leq\|a-\tilde{a}\|_{[\theta, \lambda]}+\|\tilde{a}\|_{[\theta, \lambda]} \\
& \leq m(\lambda) C_{1, \lambda}(\delta)\|a-\tilde{a}\|_{[\theta]}+\|\tilde{a}\|_{[\theta, \lambda]} \\
& \leq m(\lambda) C_{1, \lambda}(\delta) \cdot \frac{2 e^{-\rho \lambda^{2}}}{1-e^{-\rho \lambda^{2}}}\|f\|_{\mathscr{F} \infty}+C_{1, \lambda}(\rho)\|f\|_{\mathscr{F} \infty} .
\end{aligned}
$$

Taking the infimum over all $f \in \mathscr{F}\left(A_{0}, A_{1}\right)$ for which $a=f(\theta)$ we conclude that

$$
\|a\|_{[\theta, \lambda]} \leq\left(m(\lambda) \cdot \frac{2 e^{-\rho \lambda^{2}}}{1-e^{-\rho \lambda^{2}}} C_{1, \lambda}(\delta)+C_{1, \lambda}(\rho)\right)\|a\|_{[\theta]} .
$$

If we now choose $\rho=\delta=\frac{1}{\lambda}$ we see that

$$
\|a\|_{[\theta, \lambda]} \leq\left(m(\lambda) \cdot \frac{2 e^{-\lambda}}{1-e^{-\lambda}}+1\right) C_{1, \lambda}\left(\frac{1}{\lambda}\right)\|a\|_{[\theta]}
$$

for all $a \in\left[A_{0}, A_{1}\right]_{\theta}$. Since

$$
m(\lambda) \cdot \frac{2 e^{-\lambda}}{1-e^{-\lambda}}=\frac{\lambda}{2 \pi}\left(1+e^{\frac{4 \pi}{\lambda}}\right) \frac{2 e^{-\lambda}}{1-e^{-\lambda}}
$$


and

$$
C_{1, \lambda}\left(\frac{1}{\lambda}\right)=e^{\frac{1}{\lambda}}+2 e^{\frac{1}{\lambda}-0.25 \lambda} \cdot \frac{2-e^{-\lambda}}{1-e^{-\lambda}}
$$

it is easy to see that

$$
\lim _{\lambda \rightarrow \infty} m(\lambda) \cdot \frac{2 e^{-\lambda}}{1-e^{-\lambda}}=0
$$

and

$$
\lim _{\lambda \rightarrow \infty} C_{1, \lambda}\left(\frac{1}{\lambda}\right)=1
$$

hence

$$
\lim _{\lambda \rightarrow \infty}\left(m(\lambda) \cdot \frac{2 e^{-\lambda}}{1-e^{-\lambda}}+1\right) C_{1, \lambda}\left(\frac{1}{\lambda}\right)=1 .
$$

Obviously, this completes the proof of Theorem 15 .

Remark. We remark that some of our estimates in this paper are quite crude. We therefore do not claim that the expression

$$
\left(m(\lambda) \cdot \frac{2 e^{-\lambda}}{1-e^{-\lambda}}+1\right) C_{1, \lambda}\left(\frac{1}{\lambda}\right)
$$

which we obtained in the previous proof provides a tight upper bound of the asymptotic behavior of the best constant $C=C(\lambda)$ which may be written in (2.1) as $\lambda \rightarrow \infty$.

Acknowledgements: We thank Michael Cwikel for helpful discussions and for his support in the preparation of this paper.

\section{REFERENCES}

[1] C. Bennett and R. Sharpley, Interpolation of operators, Academic Press, Inc., New York, 1988.

[2] J. Bergh and J. Löfström, Interpolation spaces, An Introduction, Grundlehren der mathematische Wissenschaften 223, Springer, BerlinHeidelberg-New York, 1976.

[3] Yu. A. Brudnyi and N. Ya. Krugljak, Interpolation functors and interpolation spaces, Volume 1, North-Holland, Amsterdam 1991.

[4] A. P. Calderón, Intermediate spaces and interpolation, the complex method, Studia Math. 24 (1964), 113-190.

[5] M. Cwikel, Complex interpolation, a discrete definition and reiteration, Indiana Univ. Math. J. 27 (1978), 1005-1009.

[6] S. G. Krein, Ju. I. Petunin and E. M. Semenov, Interpolation of linear operators. Translations of Mathematical Monographs, Vol. 54, American Mathematical Society, Providence R.I., 1982.

[7] J. Peetre, Sur l'utilization des suites inconditionellement sommables dans la théorie des espaces d'interpolation. Rend. Sem. Mat. Univ. Padova 46 (1971), 173-190. 
[8] H. Triebel, Interpolation Theory, Function Spaces, Differential Operators. North-Holland, Amsterdam 1978.

Department of Mathematics, Technion - Israel Institute of TechNOLOGY, HAIFA 32000, ISRAEL

E-mail address: eliran5@tx.technion.ac.il 